\title{
Nonlinear Distortions Self-correction for Videogrammetric Measurement
}

\author{
Zhang Zhengyu \\ China Aerodynamics Research and Development \\ Center \\ Mianyang, China \\ e-mail: zzyxjd@163.com \\ Huang Xuhui \\ China Aerodynamics Research and Development \\ Center \\ Mianyang, China
}

\author{
Zhu Long \\ Information Engineering College \\ Southwest University of Science and Technology \\ Mianyang, China \\ Yin Jiang \\ China Aerodynamics Research and Development \\ Center \\ Mianyang, China
}

\begin{abstract}
- the nonlinear distortions of camera optical system produced by fabricating and assembling errors can generate the differences between real image and ideal image to some extent. As a result, a method of high accurate camera selfcalibration is presented in this paper for videogrammetric Measurement, following the coplanar principle of homologous points on different view-angle images. The coplanar condition equations with nonlinear distortion coefficients are deduced, and their numerical calculation method is established based on the generalized inverse method of least square solution to achieve the nonlinear distortion parameters of the camera. The several examples, including a calibration at $2.4 \mathrm{~m} \times 2.4 \mathrm{~m}$ transonic wind tunnel test section in China Aerodynamics Research and Development Center, have demonstrated this method is correct, and distortion coefficients can be obtained by inputting different view-angle images with more than six code points. Therefore this approach is simple and efficiency for it needn't high precise calibration objects or accurate camera movements, its cost is low.
\end{abstract}

Keywords- videogrammetric measurement;machine vision; camera calibration; distortion coefficients; coplanar condition equations

\section{INTRODUCTION}

Due to the manufacture errors of camera optical system, such as fabrication error of the lens, lens axial installation error, lens centralization error and so on, the co-line relationship of center of camera, measuring point and its image point is damaged to some degree, which reduces the precision of videogrammetric measurement ${ }^{[1-12]}$. The current camera calibration mainly based on radial distortion, eccentric distortion and thin prism distortion, and the main techniques can be divided into three categories.

(1) The methods use a high accurate $3 \mathrm{D}$ or $2 \mathrm{D}$ objects to establish co-line relationship of center of camera, a known point and its image point, then calculate the interiors parameters and external parameters of a given image. This kind of method consist of Direct Linear Transformation (DLT) ${ }^{[1-4]}$, Tsai's two-step calibration method $^{[1-4]}$, Weng's iterative method ${ }^{[1-4]}$, double plane calibration method ${ }^{[1]}$ and Z.YZhang's plane template calibration method $^{[5-6]}$. This method is easy to added the nonlinear distortion coefficients, and can achiever high accurate calibration based on co-line equations. However, if the area of calibration object is equal to or greater than $600 \mathrm{~mm} \times 800 \mathrm{~mm}$, its cost of fabrication and (constant temperature and humidity) storage will soar to several hundred thousand dollars ${ }^{[1-6]}$.

(2)The self-calibration methods follow the coplanar relationship of homologous points on different view-angle images, therefore they need not high accuracy calibration object, but they must solve nonlinear equations, where the precision of initial values determines the calibration result, so it is not robust relatively ${ }^{[10-12]}$.

(3) The calibrations based on active vision use a precise movement platform to calibrate the camera ${ }^{[10-12]}$, such as MaSongDe's two orthogonal movements of the linear method, and others improved schemes based on multi-groups of orthogonal planes orthogonal movements. In literature [12] the linear radial distortion coefficient is calculated by co-line equations, but these methods do not consider eccentric distortions and thin prism distortions, and need the initial values from movement platform. These methods are simple and can get linear solution, but need an accurate movable platform of the camera [9-10].

In this paper, a method of high accurate camera selfcalibration based on coplanar equations of homologous points is presented to obtain the nonlinear distortion parameters of the camera, which need not expensive high precision calibration objects or movable platform, therefore it is simple and its cost is low.

\section{NONLINEAR DISTORTION CORRECTIONS OF CAMERA BASED ON COPLANAR EQUATIONS}

\section{A. nonlinear optical distortion model}

Radial distortions cause the small displacement of image point along the radial direction compared with ideal image point. To ignore high order parts of radial 
distortions, the small displacement can be described as follow

$$
\left\{\begin{array}{l}
\delta x_{r}=x\left[k_{1}\left(x^{2}+y^{2}\right)+k_{2}\left(x^{2}+y^{2}\right)^{2}\right] \\
\delta y_{r}=y\left[k_{1}\left(x^{2}+y^{2}\right)+k_{2}\left(x^{2}+y^{2}\right)^{2}\right]
\end{array}\right.
$$

The optical system of the camera consists of several optical lenses. The optical axis of these lenses cannot completely be coline because of assembly errors, which produces a small eccentric displacement. To ignore high order parts of eccentric distortions, the small displacement can be described as follow

$$
\left\{\begin{array}{l}
\delta x_{d}=p_{1} x\left(3 x^{2}+y^{2}\right)+2 p_{2} x y \\
\delta y_{d}=2 p_{1} x y+p_{2} x\left(x^{2}+3 y^{2}\right)
\end{array}\right.
$$

The thin prism distortion is generated by manufacturing errors of the optical lens and CCD, which induces the small displacement of image points consisting of the radial displacement and tangential displacement. To ignore high order parts of thin prism distortion, the small displacement can be described as follow

$$
\left\{\begin{array}{l}
\delta x_{p}=s_{1}\left(x^{2}+y^{2}\right) \\
\delta y_{p}=s_{2}\left(x^{2}+y^{2}\right)
\end{array}\right.
$$

where $\mathrm{x}$ and $\mathrm{y}$ are $2 \mathrm{D}$ coordinates of the image point.

\section{B. coplanar equations including nonlinear distortion coefficients}

Given two pictures $I_{A}$ and $I_{B}$, the homologous point set is $P$, where $\forall p_{i} \in P, p_{i}=\left(p_{i}^{A}, p_{i}^{B}\right) . S_{A} u_{1} v_{1} w_{1}$ and $S_{B} u_{2} v_{2} w_{2}$ are $3 \mathrm{D}$ image space coordinate systems of $I_{A}$ and $I_{B}$ respectively. $3 \mathrm{D}$ image space coordinates of $p_{i}^{A}$ and $p_{i}^{B}$ in $S_{A} u_{1} v_{1} w_{1}$ and $S_{B} u_{2} v_{2} w_{2}$ are $\left(u_{1}, v_{1}, w_{1}\right)$ and $\left(u_{2}, v_{2}, w_{2}\right)$ respectively. $S_{1}$ and $S_{2}$ are origins of coordinate of $S_{1} u_{1} v_{1} w_{1}$ and $S_{2} u_{2} v_{2} w_{2}$ respectively. The coordinates of $S_{2}$ in $S_{1} u_{1} v_{1} w_{1}$ is $\left(b_{x}, b_{y}, b_{z}\right)$, then coplanar equations of $p_{i}^{A}$ and $p_{i}^{B}$ are

$$
F\left(p_{i}\right)=\left|\begin{array}{lll}
b_{x} & b_{y} & b_{z} \\
u_{1} & v_{1} & w_{1} \\
u_{2} & v_{2} & w_{2}
\end{array}\right|=0
$$

where

$$
\begin{gathered}
{\left[\begin{array}{l}
u_{1} \\
v_{1} \\
w_{1}
\end{array}\right]=\left[\begin{array}{c}
x_{1}^{A}+\Delta x-x_{0} \\
y_{1}^{A}+\Delta y-y_{0} \\
-f
\end{array}\right]} \\
{\left[\begin{array}{c}
u_{2} \\
v_{2} \\
w_{2}
\end{array}\right]=R_{2} \times\left[\begin{array}{c}
x_{2}^{B}+\Delta x-x_{0} \\
y_{2}^{B}+\Delta y-y_{0} \\
-f
\end{array}\right]} \\
\Delta x=\delta x_{r}+\delta x_{d}+\delta x
\end{gathered}
$$

$$
\Delta y=\delta y_{r}+\delta y_{d}+\delta y_{p}
$$

$f$ in equation(5) is focus of the camera, $\left(x_{1}^{A}, y_{1}^{A}\right)$ in equation(5) and $\left(x_{2}^{B}, y_{2}^{B}\right)$ in equation(6) are 2D image plane coordinates of $p_{i}^{A}$ and $p_{i}^{B}$ respectively. $R_{2}$ in equation(6) is the rotation matrix from $S_{2} u_{2} v_{2} w_{2}$ to $S_{A} u_{1} v_{1} w_{1}, R_{2}$ is constructed by the three angle element $(\varphi, \omega, \kappa)$. Because $b_{x}$ determines the scale of the relative orientation, as long as the total number of $P$ more than 13 , the five relative orientation elements ( $\left.b_{y}, b_{z}, \varphi, \omega, \kappa\right), 6$ distortion parameters $\left(k_{1}, k_{2}\right.$, $\left.p_{1}, p_{2}, s_{1}, s_{2}\right)$ and $\left(x_{0}, y_{0}\right)$ can be calculated by equation(4).

\section{Solution of nonlinear distortion coefficients}

In this paper, the generalized inverse method of least square solution is used to achieve the nonlinear distortion coefficients from the equation (4). Let a vector $X=$ $\left(b_{y}, b_{z}, \varphi, \omega, \kappa, k_{1}, k_{2}, p_{1}, p_{2}, s_{1}, s_{2}, x_{0}, y_{0}\right)$, the total number of $P$ is $m$, then the Jacobi matrix of equation (4) is shown as follow

$$
f(X)=\left[\begin{array}{cccc}
\frac{\partial F\left(p_{0}\right)}{\partial b_{y}} & \frac{\partial F\left(p_{0}\right)}{\partial b_{z}} & \cdots & \frac{\partial F\left(p_{0}\right)}{\partial y_{0}} \\
\frac{\partial F\left(p_{1}\right)}{\partial b_{y}} & \frac{\partial F\left(p_{1}\right)}{\partial b_{z}} & \cdots & \frac{\partial F\left(p_{1}\right)}{\partial y_{0}} \\
\cdots & \cdots & \cdots & \cdots \\
\frac{\partial F\left(p_{m}\right)}{\partial b_{y}} & \frac{\partial F\left(p_{m}\right)}{\partial b_{z}} & \cdots & \frac{\partial F\left(p_{m}\right)}{\partial y_{0}}
\end{array}\right]
$$

The iteration of equation (4) are

$$
\left\{\begin{array}{c}
X^{(k+1)}=X^{(k)}-\alpha_{k} Z^{(k)} \\
A^{(k)} Z^{(k)}=\left(F\left(p_{0}\right)^{(k)}, F\left(p_{1}\right)^{(k)}, \cdots, F\left(p_{m-1}\right)^{(k)}\right)^{T}
\end{array}\right.
$$

where $A^{(k)}$ is the Jacobin matrix of the $X^{(k)}, \alpha_{k}$ is value which makes

$$
\sum_{i=0}^{m-1}\left(F\left(p_{i}\right)^{(k)}\right)^{2}
$$

get minimum value, the rational extremum method is used to compute $\alpha_{k}$ in this paper.

\section{EXPERIMENTS AND ANALYSISES}

The calibration software used the method presented by this paper is developed by Microsoft Visual Studio $2005 \mathrm{c \#}$, and the calibration hardware consist of a DALSA ${ }^{\circledR}$ camera whose resolution is 4 million pixels and size is $17.4 \mathrm{~mm} \times 12.8 \mathrm{~mm}$, an image acquisition computer, $35 \mathrm{~mm}$ fixed-focus lens and a ruler. In $2.4 \mathrm{~m} X$ $2.4 \mathrm{~m}$ transonic wind tunnel test section of China Aerodynamics Research and Development Center, the 32 images is collected with the different view angles, as the 


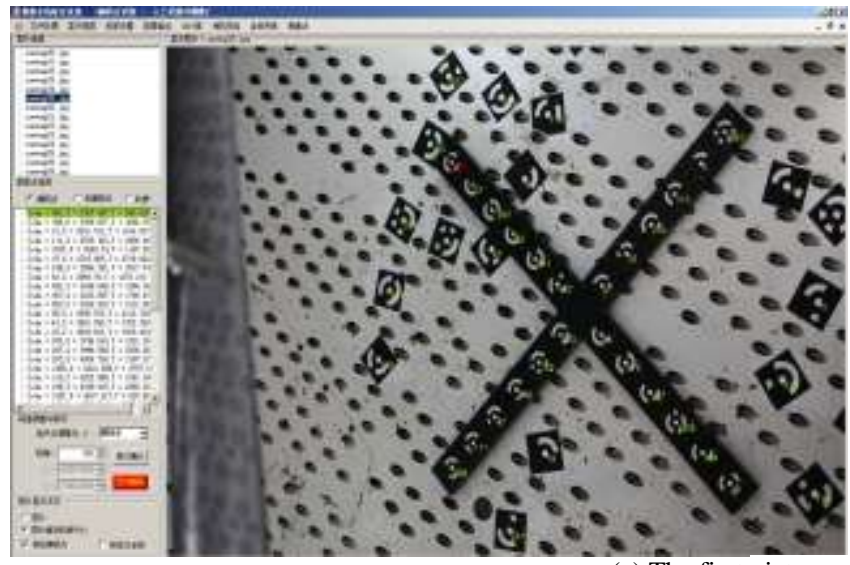

(a) The first picture

Figure 1. Interface of calibration software

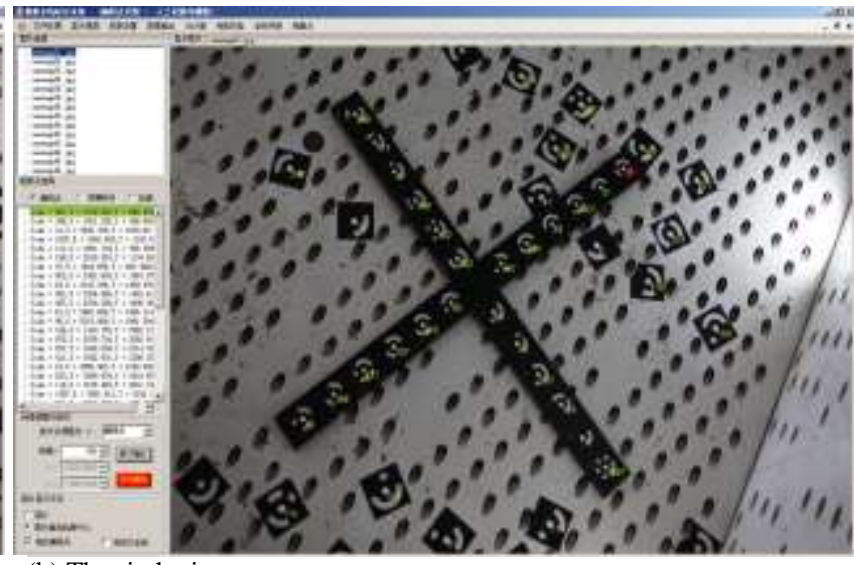

(b) The sixth picture

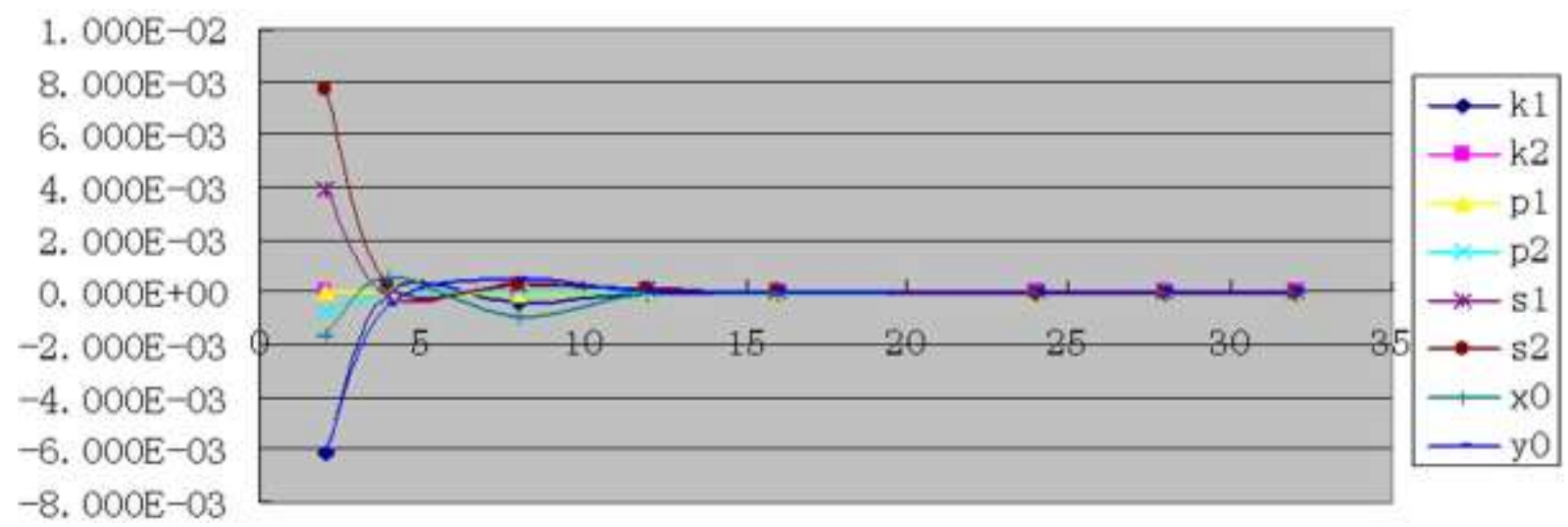

Figure 2. Relationship of nonlinear distortion coefficients and the number of images involved distortion calculation

Fig .1 shows the two images are processed in this calibration software, where the green digitals mean the numbers of coded points and the red digital means the number of a selected coded point. The six nonlinear distortion parameters are calculated from these 32 images, and are shown as table I. The relationship of six nonlinear distortion coefficients and the number of images involved distortion calculation is described by Fig .2.

Compared with the traditional calibration approach, this experiment uses the free shoots with the same camera, and the distribution density of coded points is low, therefore the accuracy nonlinear distortion coefficients cannot be obtained by 2 or 3 images. In table I, we can find the uniformity is not good on the CCD. As a result, the computed distortion parameters cannot really reflect the camera's distortion phenomenon. When the number of elected images is added from 4 to 12 , the total number of coded points taken into calculation of the distortion parameters will increase from 96 to 288 . The distribution of the coded points on CCD comes to uniformity, because the 2D image coordinates of 288 coded points are difficult to be equal for the free shoots with the same camera, therefore the calculated distortion parameters is quickly convergence. When the number of elected images is added from 16 to 32 , the total number of coded points taken into calculation of the distortion parameters will increase from 384 to 768 . The distribution uniformity of

TABLE I. RELATIONSHIP OF NONLINEAR DISTORTION COEFFICIENTS AND THE NUMBER OF IMAGES INVOLVED DISTORTION CALCULATION

\begin{tabular}{|c|c|c|c|c|c|c|c|c|}
\hline Num & $\mathbf{k 1}$ & $\mathbf{k 2}$ & $\mathbf{p 1}$ & $\mathbf{p 2}$ & $\mathbf{s 1}$ & $\mathbf{s} 2$ & $\mathbf{x 0}$ & $\mathbf{y 0}$ \\
\hline 2 & $-6.15 \mathrm{E}-03$ & $2.23 \mathrm{E}-05$ & $3.48 \mathrm{E}-05$ & $-7.29 \mathrm{E}-04$ & $3.89 \mathrm{E}-03$ & $7.69 \mathrm{E}-03$ & $-1.65 \mathrm{E}-03$ & $-6.03 \mathrm{E}-03$ \\
\hline 4 & $5.17 \mathrm{E}-05$ & $9.87 \mathrm{E}-07$ & $1.09 \mathrm{E}-05$ & $-6.62 \mathrm{E}-05$ & $-1.24 \mathrm{E}-04$ & $3.24 \mathrm{E}-04$ & $5.63 \mathrm{E}-04$ & $-4.60 \mathrm{E}-04$ \\
\hline 8 & $-4.33 \mathrm{E}-04$ & $1.72 \mathrm{E}-05$ & $-1.61 \mathrm{E}-05$ & $1.07 \mathrm{E}-05$ & $2.55 \mathrm{E}-04$ & $2.97 \mathrm{E}-04$ & $-9.63 \mathrm{E}-04$ & $4.92 \mathrm{E}-04$ \\
\hline 12 & $-9.60 \mathrm{E}-05$ & $4.53 \mathrm{E}-06$ & $-9.34 \mathrm{E}-06$ & $-3.70 \mathrm{E}-06$ & $1.62 \mathrm{E}-04$ & $1.38 \mathrm{E}-04$ & $-2.30 \mathrm{E}-05$ & $2.46 \mathrm{E}-05$ \\
\hline 16 & $-7.66 \mathrm{E}-05$ & $4.20 \mathrm{E}-06$ & $-6.39 \mathrm{E}-06$ & $1.27 \mathrm{E}-05$ & $1.77 \mathrm{E}-05$ & $-1.16 \mathrm{E}-05$ & $-5.54 \mathrm{E}-05$ & $1.98 \mathrm{E}-05$ \\
\hline 24 & $-7.74 \mathrm{E}-05$ & $3.51 \mathrm{E}-06$ & $-4.01 \mathrm{E}-06$ & $6.14 \mathrm{E}-06$ & $-5.80 \mathrm{E}-07$ & $-2.52 \mathrm{E}-05$ & $-3.49 \mathrm{E}-06$ & $-1.60 \mathrm{E}-06$ \\
\hline 28 & $-6.84 \mathrm{E}-05$ & $3.16 \mathrm{E}-06$ & $-3.74 \mathrm{E}-06$ & $5.49 \mathrm{E}-06$ & $-2.46 \mathrm{E}-06$ & $-2.07 \mathrm{E}-05$ & $-2.99 \mathrm{E}-06$ & $-6.19 \mathrm{E}-06$ \\
\hline 32 & $-6.61 \mathrm{E}-05$ & $3.12 \mathrm{E}-06$ & $-3.87 \mathrm{E}-06$ & $5.36 \mathrm{E}-06$ & $-5.32 \mathrm{E}-06$ & $-2.24 \mathrm{E}-05$ & $-3.04 \mathrm{E}-06$ & $5.93 \mathrm{E}-06$ \\
\hline
\end{tabular}

distortion parameters is relatively lager If only 2 images are elected, because only 48 coded points are taken into the distortion parameters calculation and their distribution the coded points on CCD is so good that the calculated distortion parameters have been convergence, that is, their changes are small to such a degree that increasing number 
TABLE II. 3D COORDINATES AND RELATIVE ERRORS COMPUTED BY CORRECTED 2D IMAGE COORDINATES

\begin{tabular}{|c|c|c|c|c|}
\hline Coded number & $\mathbf{X} / \mathbf{m m}$ & $\mathbf{Y} / \mathbf{m m}$ & $\mathbf{Z} / \mathbf{m m}$ & relative error \\
\hline 119 & 334.955 & 294.416 & -2106.822 & $1.7793 \mathrm{E}-04$ \\
\hline 701 & 398.965 & -6.767 & -2065.617 & $1.2465 \mathrm{E}-05$ \\
\hline 183 & 580.219 & -126.968 & -2097.507 & $9.9871 \mathrm{E}-05$ \\
\hline 627 & 652.668 & -263.456 & -2092.742 & $1.7553 \mathrm{E}-04$ \\
\hline 241 & -135.04 & 310.354 & -1982.158 & $1.6432 \mathrm{E}-04$ \\
\hline 503 & 523.163 & -315.236 & -2047.691 & $1.8133 \mathrm{E}-04$ \\
\hline 15 & -76.320 & 139.429 & -1962.116 & $1.7998 \mathrm{E}-04$ \\
\hline 116 & 415.866 & -361.518 & -2009.473 & $2.4179 \mathrm{E}-04$ \\
\hline 319 & -310.154 & -326.272 & -1822.477 & $5.7389 \mathrm{E}-04$ \\
\hline 115 & -499.115 & -217.583 & -1788.223 & $1.4627 \mathrm{E}-04$ \\
\hline 9 & 357.924 & -227.953 & -2014.479 & $1.5575 \mathrm{E}-04$ \\
\hline 47 & 308.043 & -69.977 & -2027.032 & $2.4613 \mathrm{E}-04$ \\
\hline 991 & 423.085 & 168.850 & -2106.965 & $1.3131 \mathrm{E}-04$ \\
\hline
\end{tabular}

TABLE III. 3D COORDINATES AND RELATIVE ERRORS COMPUTED BY NON-CORRECTED 2D IMAGE COORDINATES

\begin{tabular}{|c|c|c|c|c|}
\hline Coded number & $\mathbf{X} / \mathbf{m m}$ & $\mathbf{Y} / \mathbf{m m}$ & $\mathbf{Z} / \mathbf{m m}$ & relative error \\
\hline 119 & 334.847 & 294.381 & -2106.478 & $2.861 \mathrm{E}-04$ \\
\hline 701 & 398.807 & -6.764 & -2065.001 & $9.089 \mathrm{E}-04$ \\
\hline 183 & 580.333 & -126.988 & -2098.422 & $1.514 \mathrm{E}-04$ \\
\hline 627 & 653.274 & -263.711 & -2095.579 & $7.734 \mathrm{E}-04$ \\
\hline 241 & -135.059 & 310.343 & -1981.954 & $6.590 \mathrm{E}-04$ \\
\hline 503 & 523.352 & -315.383 & -2049.077 & $1.191 \mathrm{E}-03$ \\
\hline 15 & -76.290 & 139.377 & -1961.184 & $1.812 \mathrm{E}-04$ \\
\hline 116 & 415.908 & -361.605 & -2010.241 & $5.407 \mathrm{E}-04$ \\
\hline 319 & -310.482 & -326.505 & -1824.220 & $7.625 \mathrm{E}-04$ \\
\hline 115 & -501.047 & -218.279 & -1795.144 & $1.037 \mathrm{E}-03$ \\
\hline 9 & 357.812 & -227.903 & -2014.138 & $1.350 \mathrm{E}-03$ \\
\hline 47 & 307.890 & -69.948 & -2026.176 & $3.223 \mathrm{E}-04$ \\
\hline 991 & 422.963 & 168.832 & -2106.630 & $1.746 \mathrm{E}-04$ \\
\hline
\end{tabular}

TABLE IV. NONLINEAR DISTORTION COEFFICIENTS OF D80 AND 5DMARK2

\begin{tabular}{|c|c|c|c|c|c|c|}
\hline & k1 & k2 & p1 & p2 & s1 & s2 \\
\hline D80 with 35 mm fixed-focus lens & $-2.149 \mathrm{E}-05$ & $8.875 \mathrm{E}-07$ & $-4.074 \mathrm{E}-06$ & $-1.046 \mathrm{E}-06$ & $4.314 \mathrm{E}-05$ & $7.649 \mathrm{E}-06$ \\
\hline 5Dmark2 with 45 mm fixed-focus lens & $-4.864 \mathrm{E}-07$ & $7.183 \mathrm{E}-09$ & $-8.164 \mathrm{E}-08$ & $-3.090 \mathrm{E}-07$ & $1.351 \mathrm{E}-05$ & $6.611 \mathrm{E}-06$ \\
\hline
\end{tabular}

of elected images cannot have obvious effect on the values of the calculated distortion parameters.

Therefore, the total number of coded points reaches 672 at this experiment, the accurate nonlinear distortion parameters can be calculated by this method presented by this paper. Therefore, we can come to a conclusion that when the points are filled on the CCD of the camera in the calibration process, this method can get the right nonlinear distortion parameters. This method can be also used to get accurate nonlinear distortion parameters if employs the traditional calibration object with high density points.

The nonlinear distortion parameters computed by 28 images in Table 1 are used to correct the DALSA camera, then 3D coordinate of the coded points are computed and shown in Table II, while the 3D coordinate of the coded points are computed and shown in Table III with noncorrected 2D coordinates,. The relative error $\varepsilon$ can be calculated by

$$
\varepsilon=\frac{\Delta l_{\max }}{R}
$$

where $\Delta l_{\max }$ is the maximum of difference between the computed 2D image coordinates using coline equations and the 2D image coordinates of the same coded point, $R$ is the diagonal length of CCD. The relative errors of the coded points on the bottom of $2.4 \mathrm{~m} \times 2.4 \mathrm{~m}$ transonic wind tunnel test section are shown in Table II computed by the corrected $2 \mathrm{D}$ coordinates, the relative errors are shown in Table III computed by the no-corrected 2D coordinates. Taking the 47 coded point for example, its relative error drops to $1.5575 \mathrm{E}-04$ from $1.350 \mathrm{E}-03$, therefore, this method is effective.

The digital SLR camera Nikon D80 (24 mm fixedfocus lens) and Canon 5D MarkII (45 mm fixed-focus lens) are also calibrated by this method, their nonlinear distortion coefficients are shown in Table IV.

\section{CONCLUSIONS}

A method of high accurate camera self-calibration using the homologous points on different view-angle images is presented in this paper. The coplanar condition equations including six nonlinear distortion coefficients are deduced, and their numerical calculation method is 
established based on the generalized inverse method of least square solution to achieve six nonlinear distortion parameters of the camera. Three camera calibration experiments have been verified that this method can realize the camera nonlinear distortion self-calibration, and it needn't high precise calibration objects or accurate camera movable platform, its cost is low.

\section{ACKNOWLEDGMENT}

This study is supported by National Nature Science Foundation of China (51075385) and innovation group of High Speed Aerodynamics Research Institute of China Aerodynamics Research and Development Center.

\section{REFERENCES}

[1] YANG Biwu, GUO Xiaosong. Overview of Nonlinear Distortion Correction of Camera Lens [J]. Journal of Image and Graphics, 2005, 10(3):269-274.

[2] Xu Liyan. Multi-View Connection Camera Calibration Algorithm[D]. Jinan: Shangdong University, 2008

[3] Ahmed Moumen, Farag Aly. Nonmetric calibration of camera lens distortion: Differential methods and robust estimation [J]. IEEE Transactions on Image Processing, 2005, 14 (8):1215 1230.

[4] HU Ya-jiang. A Simple Method for Calculating Radial Distortion Parameter of Camera[J]. GEOMATICS \& SPATIAL INFORMATION TECHNOLOGY, 2009, 32(5) :182-184
[5] Z.YZhang. Camera Calibration with One-Dimensional Objects IEEE Trans On PatternAnalysisAnd Machine Intellingence, 2004 7(26): 892-8990.

[6] Z.Zhang. A flexible new technique for camera calibration Pattern Analysis and Machine Intelligence. IEEE Transactions, 2002, 11(22) : 1330-1334.

[7] Wu Chaofu. New Active Vision Based Camera Self-Calibration Technique[J]. ACTA AUTOMATICA SINICA 2001, 27(6):736746.

[8] YU Hong, CHEN Hui. New camera calibration algorithm based on two perpendicular intersect lines[J]. Computer Applications,2006, 26(1):163-164:

[9] [9] Huo Ju,Yang Wei,Yang Ming. A Self-Calibration Technique Based on the Geometry Property of the Vanish Point [J]. Acta Optica Sinica, 2010, 30(2): 465 472.

[10] A. Habed, B. Boufama. Camera self-calibration from bivariate polynomials derived from Kruppa's equations [J]. Pattern Recognition, 2008, 41(8): 2484-2492.

[11] Y. Li, Y. S. Hung, L. Sukhan. A stratified self-calibration method for circular motion in spite of varying intrinsic parameters [J]. Image and Vision Computing, 2008, 26(6): 731-739.

[12] G. Yang, F. Zheng, X. H. Xu. An Analytic Solution of a Linear Camera Self-Calibration[C].Proceedings of the 6th World Congress on Intelligent Control and Automation, 2006, 2:99309934. 